\title{
Scaling guidelines of a soliton-based power limiter for 2R-optical regeneration applications
}

\author{
J. Fatome and C. Finot
}

\begin{abstract}
In this work, we report scaling rules for the design of an all-fibered soliton-based power limiter for reamplification and reshaping (2R) regeneration process. In particular, we propose general guidelines to fix the optimum fiber length and initial power of the regenerator. We quantitatively point out the optical power limiting effect of the device enabling a significant reduction of the amplitude jitter of a degraded signal. Influence of the initial level of amplitude jitter is discussed and the results are compared with a self-phase modulation-based configuration working in the normal dispersion regime. Realistic numerical simulations in the context of $160 \mathrm{Gbit} / \mathrm{s}$ signals confirm that an efficient improvement of the signal quality can be achieved by means of such a device.
\end{abstract}

Index Terms - Optical regeneration, Nonlinear optics, Optical fiber, Optical Telecommunication.

\section{INTRODUCTION}

$\mathrm{W}$ ith the development of long haul photonic networks working at high repetition rates $(40 \mathrm{Gbit} / \mathrm{s}$ and beyond), performing an all-optical regeneration of the transmission signal has become of a great interest to combat the cumulative impairments occurring during the signal propagation and to overcome the bandwidth limitations of the current electronic devices [1]. Indeed, during its propagation, the signal undergoes various degradations such as amplified spontaneous emission noise accumulation, chromatic dispersion as well as intra-channel non-linear effects. Combination of all those effects translates into degradation of the extinction ratio, into timing jitter [2] or into detrimental amplitude jitter on the "one"-bit level of the signal [3]. This last impairment can be partly overcome using $2 \mathrm{R}$ regeneration (Reamplification and Reshaping), in which a device performs optical limiting function. This can be achieved by using various optical methods taking advantage of the instantaneous Kerr nonlinear response of optical fibers : non-linear optical loop mirrors (NOLMs) [4], four-wave mixing [5], or self-phase modulation (SPM) in normally [6] or anomalous [7] dispersive fibers (the two latest methods being known as "Mamyshev" (MR) and "spectrally filtered solitons" respectively). Whereas many works, both experimental [6,

Manuscript received November XX, 2009.

This work was supported by the Agence National de la Recherche (FUTUR - ANR-06-TCOM-016 - and PERSYST II - ANR-07-TCOM014 projects.)

J. Fatome and C. Finot are with the laboratoire Interdisciplinaire Carnot de Bourgogne, UMR 5209 CNRS - Université de Bourgogne, 9 Av. A. Savary, BP 47870, 21078 Dijon Cedex, France (e-mails: julien.fatome@u-bourgogne.fr and christophe.finot@u-bourgogne.fr).
8-13] and theoretical (see [9, 12-15] and reference therein), have recently dealt with the possibilities of MR devices, much less studies have investigated the performances of soliton-based regenerators [1, 16-20].

In the present contribution, we try to address this lack by proposing some general theoretical guidelines to optimize the choice of the device parameters. After describing our model, we will detail some general design rules that enable a fast and reliable choice of optimized parameters. The influence of the initial level of amplitude jitter will also be investigated. We will discuss several significant conclusions that can be drawn as well as the advantages and drawbacks of using a soliton-based configuration compared to the now wide spread MR technique. Finally, we will illustrate the design rules by presenting numerical simulations dealing with a signal at a repetition rate of $160 \mathrm{Gbit} / \mathrm{s}$ and with a duty cycle of $25 \%$.

\section{TYPICAL SET-UP AND MODEL}

\section{A. Typical set-up}

A typical set-up of the regenerator is sketched in Fig. 1. The incoming pulsed signal is first amplified by an Erbium Doped Fiber Amplifier (EDFA) in order to reach the adequate peak power $P_{i n}$. We consider in this section the evolution of chirp-free Gaussian pulses with a full width at half maximum (FWHM) linked to the half-width $T_{0}$ at 1/e by $T_{F W H M}=2 \sqrt{\ln 2} T_{0}$. Note that despite the fact that its name indicates solitons, the use of our regenerator is absolutely not restricted to initial soliton pulses.

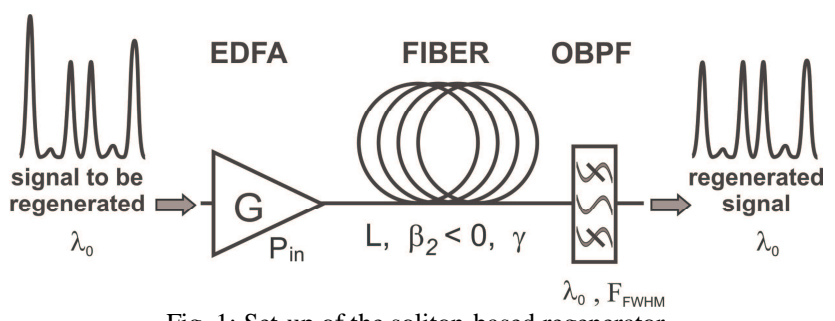

Fig. 1: Set-up of the soliton-based.regenerator

During the propagation in an anomalous dispersion fiber, the degraded signal experiences self-phase modulation (SPM) providing spectral broadening (Fig. 2(a)), especially in the spectral wings of the pulse where the extra-energy compared to the fundamental soliton is rejected. In the 
temporal domain, the pulse exhibits a stage of temporal compression (Fig. 2(b), solid black line) [21]. The resulting signal is then filtered by means of a Gaussian optical bandpass filter (OBPF) having the same central wavelength as the signal. The spectral width of the filter is directly related to the input pulse duration (for Gaussian pulses, $\left.F_{F W H M}=0.441 / T_{F W H M}\right)$. After filtering, the pulse exhibits a temporal shape close to the input pulse (Fig 2(b), dotted grey line), is nearly transformed limited and does not exhibit any substructures.
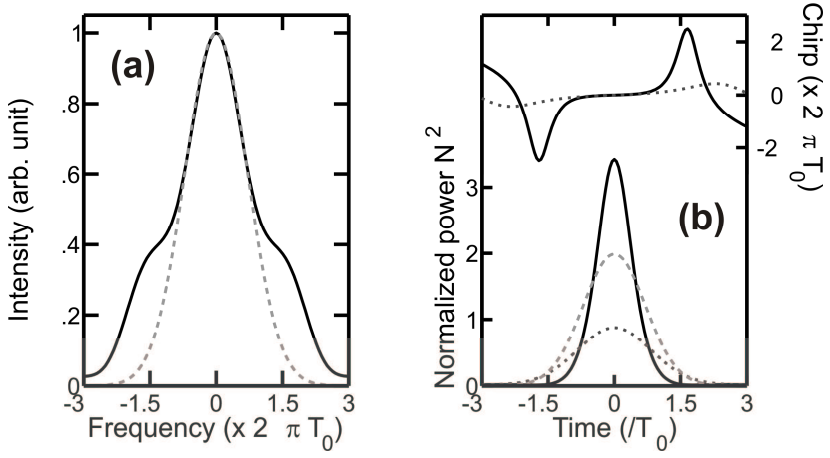

Fig. 2: (a) Spectrum at the output of the fiber (solid black line) and OBPF corresponding to the initial pulse spectrum (dashed grey line) (b) Output temporal intensity and chirp profiles obtained after propagation (solid black line) and after spectral filtering (dotted grey line) are compared to the input temporal intensity profile (dashed line). Results are obtained for an initial normalized input power $N_{i n}=1.5$ and a propagation length $\xi_{L}$ $=1.41$ (see section $\mathrm{B}$ for the definition of the normalized quantities).

Interestingly, our lumped regenerator can also be partly understood in view of the various studies carried out in early nineties in the context of soliton transmission control where OBPFs were periodically inserted all along the optical link [22, 23]. Note however that the main target of this optical scheme was to limit the temporal jitter linked to the Gordon-Haus effect [2].

\section{B. Normalized numerical model}

In order to assess the performance of the architecture, we have simulated the propagation of a single chirp-free Gaussian pulse in the proposed regenerator. The longitudinal evolution of the complex electric field $\psi(z, t)$ can be modeled using the well-known non-linear Schrödinger equation (NLSE) including the second order dispersion $\beta_{2}$ and the non-linear coefficient $\gamma$ [21] :

$$
i \frac{\partial \psi}{\partial z}=\frac{\beta_{2}}{2} \frac{\partial^{2} \psi}{\partial t^{2}}-\gamma|\psi|^{2} \psi .
$$

As we have considered dispersion flattened fibers or fibers with a high $\beta_{2} / \beta_{3}$ ratio, we have neglected for the sake of simplicity the influence of the third order dispersion (TOD) $\beta_{3}$. Given the reduced fiber length required (typically less than one kilometer) and the low loss level of available fibers (below $0.6 \mathrm{~dB} / \mathrm{km}$ ), we have also neglected the consequences of the linear losses.

In order to make our results applicable to a wide range of experimental situations (i.e. for different input temporal widths as well as for various nonlinear and dispersive fiber parameters), it is extremely beneficial to transform Eq. (1) into the following canonical form [21]:

$$
i \frac{\partial u}{\partial \xi}=-\frac{1}{2} \frac{\partial^{2} u}{\partial \tau^{2}}-|u|^{2} u,
$$

where $u, \tau$ and $\xi$ are the normalized parameters defined as

$$
\begin{gathered}
u(\xi, \tau)=N_{\text {in }} \quad U, \quad U(\xi, \tau)=\frac{\psi}{\sqrt{P_{i n}}}, \\
\tau=\frac{T}{T_{0}}, \quad \xi=\frac{z}{L_{D}} .
\end{gathered}
$$

$L_{D}, N_{\text {in }}, N_{\text {out }}$ and $\xi_{L}$ are the dispersion length, the soliton orders, and the normalized fiber length defined as follows:

$$
\begin{gathered}
L_{D}=\frac{T_{0}^{2}}{\left|\beta_{2}\right|}, \quad N_{\text {in }}=\sqrt{\gamma P_{\text {in }} L_{D}}, \\
N_{\text {out }}=\sqrt{\gamma P_{\text {out }} L_{D}}, \quad \xi_{L}=\frac{L}{L_{D}} .
\end{gathered}
$$

with $P_{\text {in }}$ and $P_{\text {out }}$ the input and output peak powers respectively. Similarly, we can define a normalized quantity $N(\tau)$ based on the instantaneous peak power $P(t)$ :

$$
N(\tau)=\sqrt{\gamma L_{D} P(t)} .
$$

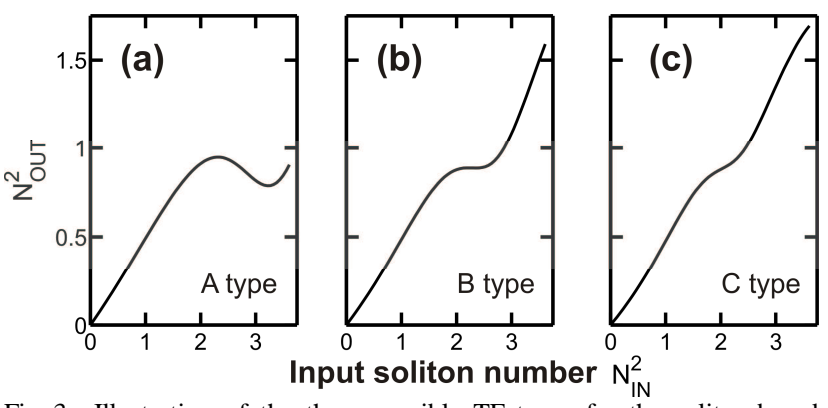

Fig. 3: Illustration of the three possible TF types for the soliton-based regenerator : rise and fall evolution (A), locally flat evolution (B) and monotonous variation (C). Results are obtained for normalized propagation distance $\xi_{L}$ of $1,1.41$ and 1.8 respectively.

\section{REGENERATION PERFORMANCE AND OPTICAL LIMITING PROPERTIES}

\section{A. Transfer functions and optical limiting properties}

The ability of the device to efficiently perform the expected optical limiting function can be assessed by the widely used transfer function (TF) which links the input and output peak powers (or equivalently with our normalized parameters $N_{\text {in }}^{2}$ and $\left.N_{\text {out }}^{2}: N_{\text {out }}^{2}=R\left(N_{\text {in }}^{2}\right)\right)$. Depending on the fiber length $\xi_{L}$, we have identified that the interplay between nonlinearity and chromatic dispersion leads to three possible regimes for the $\mathrm{TF}$, as graphically illustrated in Fig. 3. For $\xi_{L}<1.41$, the TF is characterized by a rise and fall evolution (regime 'A'). On the contrary for $\xi_{L}>1.41$, the TF becomes purely monotonous (regime ' $\mathrm{C}$ '), which should obviously be banned for the purpose of regeneration. The most interesting behavior is observed for $\xi_{L}=1.41$ where the TF exhibits a locally flat region called plateau (regime 'B'). 
In order to better quantify the limiting properties of the optical device, we have systematically computed the output amplitude jitter (defined here as the peak-to-peak value of the peak power variations of the regenerated signal). We have considered an input signal with peak-to-peak fluctuations $\Delta \varepsilon$ of the peak power. We have first considered $\Delta \varepsilon= \pm 7.5 \% N_{i n, 0}^{2} \quad$ (in other words, the peak-powers of initial pulses $N_{i n}^{2}$ vary from $0.925 N_{i n, 0}^{2}$ to $1.075 N_{i n, 0}^{2}$, $N_{i n, 0}^{2}$ being the working power of the regenerator). Results are shown in Fig. 4 on a two-dimension map that enables us to quickly determine the optimum set of parameters. We have also superposed the points corresponding to local extrema of the TF. Let us note that for the MR design, a more complex map was required since an additional parameter, i.e. the spectral offset of the OBPF, had to be taken into account [15].

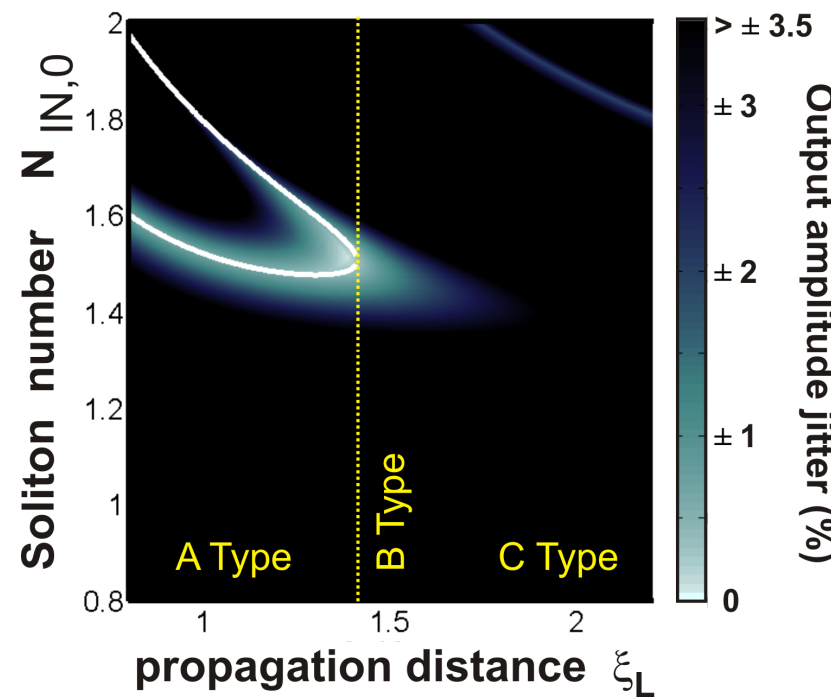

Fig. 4: Output signal amplitude jitter as a function of the normalized fiber length and input peak power for an input relative amplitude jitter of \pm 7.5 $\%$. The solid white lines are the local extrema of the TF.

From the map of Fig. 4, we can make out several points. First, the different types of TF are readily apparent: regime A corresponds to the existence of two local extrema which get closer and closer when the propagation length increases. When the two branches join, a stationary point of inflexion is obtained for a precise distance and initial power (Type B) that we have found to be $\xi_{L}=1.41$ and $N_{i n, 0}=1.5$. For longer lengths, no local extremum can be found (Type C). Intense numerical simulations have enable to confirm that for a $\Delta \varepsilon= \pm 7.5 \% N_{i n, 0}^{2}$ input amplitude jitter, optimum reduction of the peak power fluctuations was obtained for a point extremely close to those predictions $\left(\xi_{L}=1.38\right.$ and $N_{i n, 0}=1.51$ ), just before the B type point. Power equalization has been very efficient, with output fluctuations below $0.2 \%$. This also confirms that the B-shape is a priori very well suited for regeneration.

\section{B. Influence of the level of initial amplitude jitter}

In order to accommodate the significant amplitude jitters that develop after hundreds of kilometers of propagation, it is also of interest to consider larger initial amplitude fluctuations, i.e. relative amplitude jitter of $\pm 15 \%$ and $\pm 30 \%$. The corresponding 2D-maps are depicted in Figs. 5(a) and 5(b) respectively and optimal sets of parameters correspond to light areas.

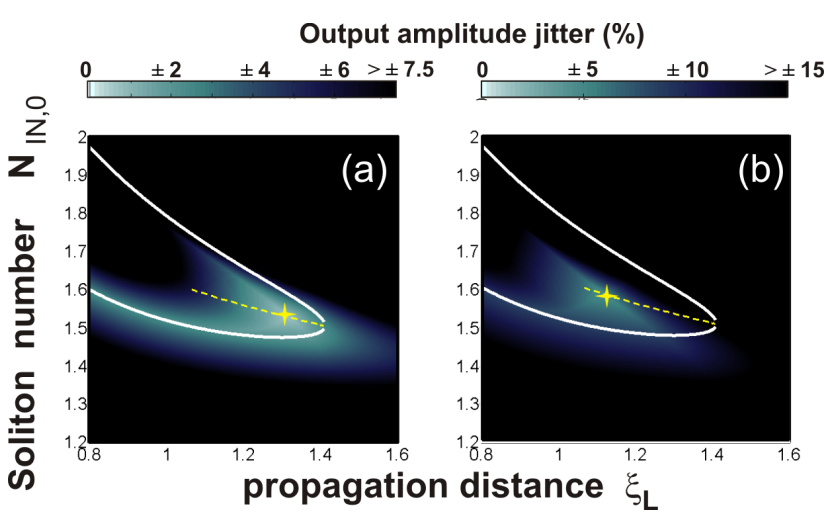

Fig. 5: Output signal peak-to-peak amplitude jitter as a function of the normalized fiber length and input peak power for an input amplitude jitter of $\pm 15 \%$ and $\pm 30 \%$ (subplots (a) and (b) respectively). The white solid lines are the local extrema of the TF. The crosses represent the optimum set of parameter where minimal output jitter is performed. The dashed line corresponds to the optimum parameters found for different initial amplitude jitters as predicted by Fig. 6(b).

From simulations of Figs. 4 and 5, we can conclude that whereas using a C-type TF definitively does not provide any improvement; it can be worthy to use an A-type TF for which the non-optimum point does not translate into severe penalty. It seems on the contrary that such a kind of TF offers a range of working powers larger that B-type, which can constitute an attractive advantage in the practical design of the device. Such a feature can be quite easily understood by considering the following Taylor expansion of the TF $R\left(N_{i n}^{2}\right)$ around the working power $N_{i n, 0}^{2}$ :

$$
\begin{aligned}
& N_{\text {out }}^{2}\left(N_{i n, 0}^{2}+\mathcal{E}\right)=R\left(N_{i n, 0}^{2}\right)+R^{\prime}\left(N_{i n, 0}^{2}\right) \mathcal{E} \\
& \quad+R^{\prime \prime}\left(N_{i n, 0}^{2}\right) \frac{\mathcal{E}^{2}}{2}+R^{\prime \prime \prime}\left(N_{i n, 0}^{2}\right) \frac{\mathcal{E}^{3}}{6}+\ldots
\end{aligned}
$$

with $R$ ', $R$ ', $R$,"' being the first, second and third order derivatives of $\mathrm{R}\left(N_{i n}^{2}\right)$. The relative output jitter $J$ resulting from an initial amplitude jitter $\Delta \varepsilon$ can easily be estimated by :

$$
J(\Delta \mathcal{E})=\frac{\max \left(\left|N_{\text {out }}^{2}\left(N_{\text {in }, 0}^{2}+\varepsilon_{1}\right)-N_{\text {out }}^{2}\left(N_{\text {in }, 0}^{2}+\varepsilon_{2}\right)\right|\right)}{N_{\text {out }}^{2}\left(N_{\text {in }, 0}^{2}\right)}(7)
$$

with $\varepsilon_{1}$ and $\varepsilon_{2}$ being in the interval [- $-\Delta \varepsilon \Delta \varepsilon$.

For very low initial jitters a Taylor expansion up to the first order is sufficient, which imposes to $R^{\prime}\left(N_{i n, 0}^{2}\right)=0$ to cancel the output amplitude jitter. Therefore, it is possible to work close to the extrema of the TF, as can be seen in Fig. 4 with clear jitter improvement in the vicinity of the white line. Let us however note that considering such a reduced level of initial fluctuations presents little interest : the high quality signal does not require at this stage any optical regeneration. This is much more interesting to 
consider higher fluctuations (such as $\Delta \varepsilon= \pm 7.5 \% \quad N_{i n, 0}^{2}$ ), which imposes to take into account the first two terms of the Taylor expansion. Consequently, in order to get the best amplitude jitter improvement, one has to work with $R^{\prime}\left(N_{i n, 0}^{2}\right)=R^{\prime \prime}\left(N_{i n, 0}^{2}\right)=0$. Stated differently, one has to work on a stationary point of inflexion, which defines the Bregime.

If now fluctuations with stronger amplitude are considered ( such as $\Delta \mathcal{E}= \pm 30 \% N_{i n, 0}^{2}$ ), development up to the third order would lead to work with $R^{\prime}\left(N_{i n, 0}^{2}\right)=$ $R^{\prime \prime}\left(N_{i n, 0}^{2}\right)=R^{\prime \prime \prime}\left(N_{i n, 0}^{2}\right)=0$. However such a situation is not physically observed in our regenerator, the device presenting at the stationary inflexion point $R^{\prime \prime \prime}\left(N_{i n, 0}^{2}\right)>0$.

But we can fortunately note that output amplitude jitter can be optimized by working in the A-regime, between the two extrema (Fig. 5). Indeed, for such a region, it is possible to find working powers for which the non-zero contribution of $R$ ' (which is of negative sign) can be partly compensated by the influence of $R$ ', and $R$,', Such a physical situation is illustrated in Fig. 6(a) for $\pm 30 \%$ jitter where we compare operating at the stationary inflexion point and at the point $\left(N_{i n, 0}=1.58, \xi_{L}=1.12\right):$ resulting output amplitude jitter are $\pm 15 \%$ and $\pm 5 \%$ respectively. In both cases, the amplitude fluctuations have been decreased but we can clearly see that the second set of parameters leads to better performances.
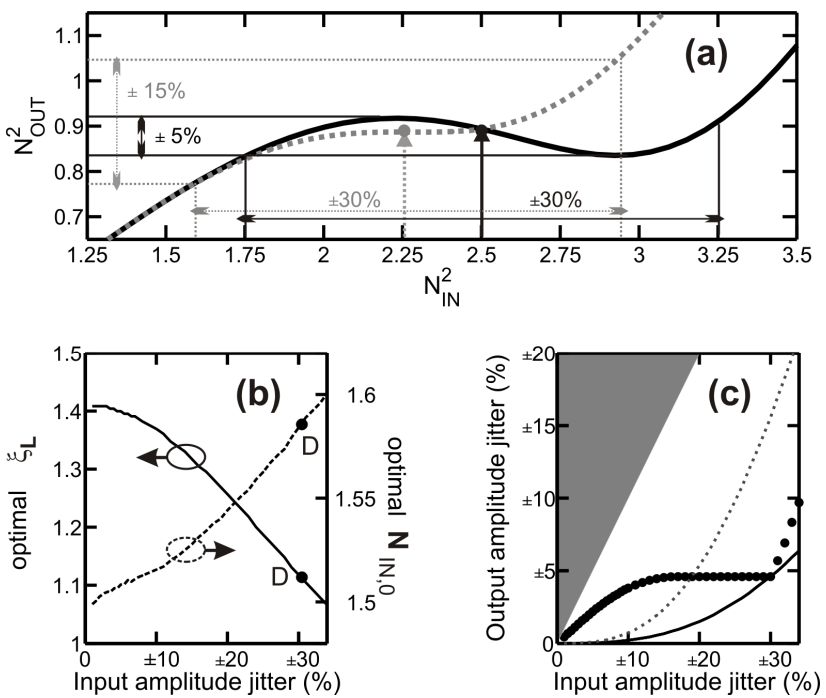

Fig. 6: (a) Illustration of output amplitude jitter improvement by using an A-type curve. Results obtained with a B-type regenerator (grey dotes curves) are compared with results based on a regenerator operating at $N_{i n, 0}=1.58, \xi_{L}=1.12$ (solid black line). Initial amplitude jitter is $\pm 30 \%$ (b) Evolution of the working parameters $\xi_{L}$ and $N_{i n, 0}$ as a function of the input amplitude jitter (solid and dashed lines respectively). (c) Evolution of the output amplitude jitter when working at the optimum parameters described in Fig. 6(b) and at the B-regime (solid black and dotted grey lines respectively). The full circles are the results for the working point $\left(N_{i n, 0}=1.58, \xi_{L}=1.12\right)$. The shaded area outlines the zone where any improvement of the amplitude jitter is observed.

A more systematic investigation of the impact of the initial jitter on working points and performance has been carried out. Results are plotted in Fig. 6 (b-c) (see also the dashed lines in Figs. 5). Higher initial amplitude jitter leads to a lower regenerator length and a higher working power. The operating point therefore goes progressively away from the B-regime and moves between the two TF extrema, especially for initial amplitude fluctuations above $\pm 10 \%$. Working in the A-regime can indeed provide significant improvements.

Those different conclusions are significant in several views. First, let us stress that the present analysis based on $\mathrm{TF}$ is not restricted to soliton-based regenerator and can be easily extended to other SPM-based regenerators. Then, depending on the place of the regenerator in the transmission line, the regenerator would have to handle different levels of amplitude jitter. We can indeed expect that at the end of a transmission line before the receiver, the jitter will be much higher that at the mid-stage of the propagation. As the result, the working parameters should be ideally adapted in each situation. However, for practical reasons, the physical properties of the regenerator (fiber length and working power) are usually fixed so that tradeoffs have to be found to ensure regeneration over a wide range of operating conditions. Example of such trade-off is illustrated on Fig. 6(c) (curve with black circles) for which we have arbitrarily considered the fixed working point $\left(N_{i n, 0}=1.58, \xi_{L}=1.12\right.$, corresponding to point D in Fig. 6(b)). Intermediate performance are achieved : even if optical limiting of the output is observed for a wide range of input jitter, we can notice that for jitters below $\pm 20 \%$, performance are below the performance that can be achieved with a B-type regenerator. Much better performance is only reached for input jitter higher that $\pm 20 \%$. The shape of the curve that is obtained can be understood with the help of the TF plotted in Fig. 6(a) (solid black line). For low jitters ( $\Delta \varepsilon$ below $\pm 10 \%)$, output jitter is directly proportional to the input jitter. Indeed, for such a range of fluctuations, the $\mathrm{TF}$ is well fitted by a linear decrease. For initial jitters up to $\pm 30 \%$, the output jitter then remains constant : the output values fall in a fixed interval defined by the two local extrema of the TF and the performance of the device does not depend on the signal quality. Given the initial choice of the working parameter, at $\pm 30 \%$ of initial jitter, the curve corresponds to the solid black line describing the optimum regeneration. For higher values of initial jitters, from the shape of the $\mathrm{TF}$, we can infer that any increase of $\Delta \varepsilon$ will directly translate into additional output jitter, which is confirmed by the linear increase observed in Fig. 6(c).

\section{Influence of the initial pulse properties}

The previous discussion relies on the consideration of a single Gaussian pulse with a fixed temporal width $T_{0}$. However, it is known that in self-phase modulation based regenerators and contrary to NOLM devices, the behavior of the device depends both on the initial peak-power as well as on the temporal width $[9,15]$. It is therefore of interest to consider how the working points are affected. By considering initial temporal durations varying by $\pm 15 \%$ (energy of the pulse as well as all the other pulse parameters 
are kept constant), we have found that the set of parameter predicting the B-regime $\xi_{L}$ and $N_{i n, 0}$ vary by $20 \%$ and $5 \%$ respectively (this is directly derived from the definitions of the dispersion length and soliton number by a simple variationnal calculus). Seen differently, for a given initial amplitude jitter of $\pm 7.5 \%$, if we keep the working point as defined by the previous investigation $\left(N_{i n, 0}=1.41\right.$, $\left.\xi_{L}=1.5\right)$, the output jitter is increased up to $\pm 4 \%$ (Fig. 7(a), dotted grey line). The device seems more tolerant regarding higher pulse durations than for lower pulse durations. Let us now consider the case when the initial signal is affected by a higher level of amplitude jitter ( $\Delta \varepsilon=$ $\pm 30 \%$ and operating point $N_{i n, 0}=1.58, \xi_{L}=1.12$ ). The impact of initial pulse duration fluctuations translates into output amplitude jitter which is increased up to $\pm 12 \%$, which is more than twofold the output jitter recorded in absence of initial pulse duration fluctuations.
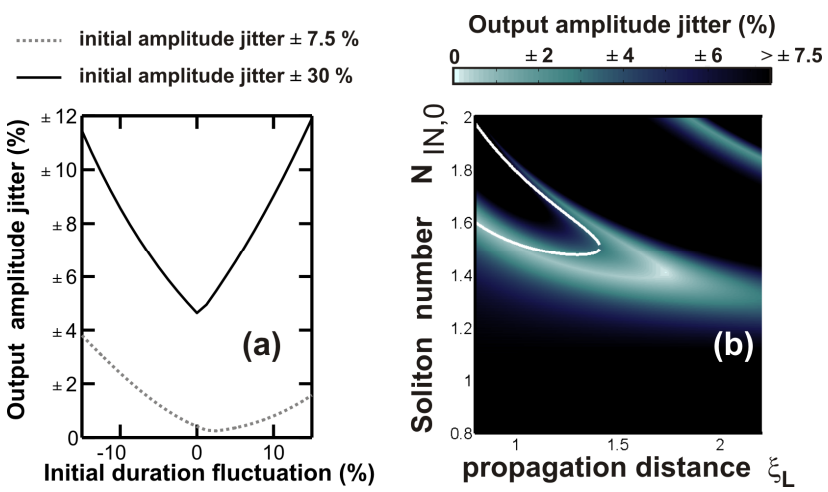

Fig. 7: (a) Output amplitude jitters as a function of initial pulse width fluctuations for a device designed for initial amplitude jitters of $\pm 7.5 \%$ and $\pm 30 \%$ (dotted grey line and solid black line, respectively). The working parameters are $\left(N_{i n, 0}=1.41, \xi_{L}=1.5\right)$ and $\left(N_{i n, 0}=1.58, \xi_{L}=\right.$ 1.12) respectively. (b) Output signal amplitude jitter as a function of the fiber length and input peak power for an input relative amplitude jitter of $\pm 7.5 \%$ of a sech pulses. The solid white lines are the local extrema of the $\mathrm{TF}$ as obtained in Fig. 4 for Gaussian initial pulses.

Another question that may arise is the tolerance of the device to different pulse shape. Indeed, we have considered an hyperbolic secant (sech) initial pulse instead of a Gaussian pulse (both pulses have the same peak power and FWHM). Performances of the device for an initial amplitude jitter of $\pm 7.5 \%$ are summarized in Fig. 7(b). As can be seen, the change of initial pulse shape has strongly affected the location of the optimum operating point so that if one keeps the operating point previously obtained for Gaussian pulse (Fig. 4), poor performance will be obtained. This can be explained mainly by two factors. First, a sech shape does not introduce the same amount of SPM as a Gaussian pulse. Then, the filter that we use at the output has a spectral width and shape defined based on a Gaussian initial pulse, which is not fully appropriate for a sech pulse shape (with a different spectral extension). Additional simulations have confirmed that increasing the initial amplitude lead to the same trends as for the Gaussian pulse, i.e. decreasing the required fiber length and increasing the initial peak-power. As a consequence, for high jitters such as $\pm 30 \%$, it becomes possible to fall in the range of previously defined operating conditions (close to the B-type working point for Gaussian pulse).

\section{IMPACT OF THE REGENERATOR ON OTHER PARAMETERS}

As it has been shown in previous papers $[14,15]$, the impact of an optical $2 \mathrm{R}$ regenerator based on self-phase modulation is not restricted to amplitude jitter reduction, indeed other pulse parameters are affected by the nonlinear propagation followed by spectral filtering. We have carried out a similar analysis as the one reported in $[14,15]$ where several points have been investigated in the context of MR and we compare the two configurations. Note that in a preceding article, Matsumoto made a similar comparison, but in the context of cascaded in-line regenerators combined with a resynchronization function [24]. The study we detail here focuses exclusively on the use of a single self-phase modulation based regenerator.

\section{A. Power efficiency and extinction ratio}

One of the main conclusions of the previous section is that working with an initial power close to $N_{i n, 0}=1.5$ gives accurate amplitude jitter reduction. This has to be compared to MR set-up requirements which usually involve $N_{i n, 0}>8$ for convenient operation. This represents at least a 14-dB reduction in the required operating average power. Let us note that despite the fact that the soliton regenerator requires a longer fiber length (1.41 $L_{D}$ instead of 0.25 $L_{D}$ for the MR device), the product of the power by the length is still in favor of the soliton-like regenerator. This can be partly attributed to the fact that in the anomalous dispersion regime of propagation, due to solitonic compression, the peak-power of the pulse increases, whereas in the normal regime, the peak power progressively decreases [25]. This reduction of the product power by length is also favorable in order to avoid the experimental problem of Brillouin backscattering which has been found critical in Mamyshev configurations [11, 12].

The central filtering combined with a relatively modest spectral broadening leads to a very good energy yield with more than $40 \%$ of the initial energy remaining in the filtered signal, which has to be compared with a typical yield of $10 \%$ for MR configurations [15]. The solitonbased device is therefore much power efficient if only optical limiting is targeted.

We have also investigated how the soliton-like regenerator affects the extinction ratio of the signal (defined as the ratio between the peak powers of a 'zero' and 'one' pulse [15]). For an input extinction ratio of $-10 \mathrm{~dB}$, the resulting extinction ratio after regeneration is $-9 \mathrm{~dB}$. Indeed, the transmission through the regenerator for low powers is close to linear. Let us add that a continuous background launched in the optical limiter will suffer from an excess gain compared to pulsed signal [22] (due to the output filter, the pulsed signal will experience selective losses that are more than twice the losses undergone by a continuous 
wave). In other words, our device does not provide any enhancement in space bit slots and on the contrary, it could even slightly degrade this point. Therefore, it stresses the need to use an additional optical device especially if cascading of the regeneration process is required $[1,18$, 19].

\section{B. Temporal properties of the regenerated signal}

A SPM-based regenerator also impacts the temporal properties of the output pulses. From Fig. 8, we can make out that the temporal width after the regenerator for the optimum working point is comparable with the initial temporal width. More precisely, the output pulses are around $10 \%$ longer than the initial ones. This broadening is mainly attributed to the intrinsic spectral narrowing occurring during the spectral filtering process and leading in the temporal domain to longer pulses. It is therefore anticipated that reduced temporal broadening could be achieved by an appropriate choice of filter properties (spectral width and shape). Let us note that compared to Mamyshev architecture [15], the output pulses exhibit a very low level of residual chirp (see Fig. 1(b)). There is furthermore a slight power-dependence of this data, which may lead to some temporal width fluctuations at the output of the regenerator. This temporal dependence has however been found lower that in the case of a MR where the timedependence has been found to be maximized at the operating point $[13,14]$.

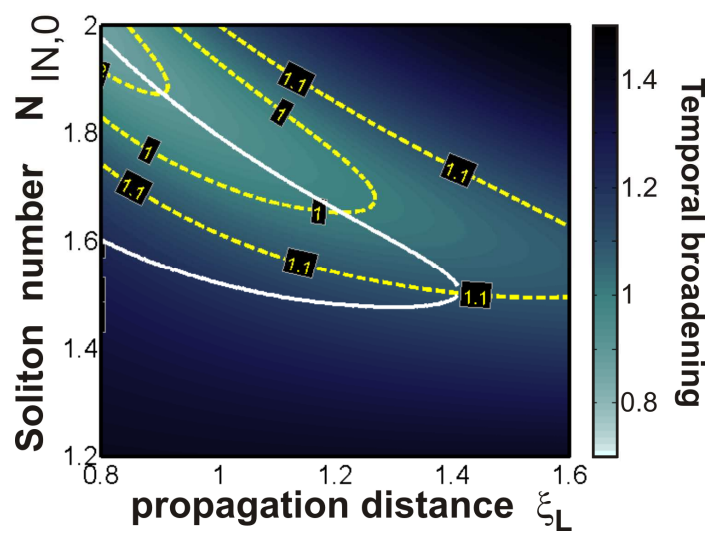

Fig. 8: Temporal broadening factor as a function of the fiber length and input peak power. The broadening is defined by the ratio between the durations at the input and output of the regenerator. The white solid lines are the local extrema of the TF. The dashed lines are contour level lines.

An additional potential drawback that has been highlighted in MR configurations is that the offset spectral filtering induces a time-delay which can be potentially translated into detrimental temporal jitter [14]. As our configuration is based on central filtering, none of such a negative point is coming out and thus, the filtered pulse is not delayed compared to the initial pulse as can be previously seen on Fig. 2(b).

Finally, another aspect we stress is that the dynamics undergone in the anomalous dispersive fiber of the soliton regenerator leads to a soliton-like compression [21, 26], which is fundamentally different from the continuous temporal broadening undergone in the normal regime [25]. As an important consequence, we can expect that no nonlinear interaction between adjacent pulses of a sequence will occur, so that detrimental patterning effects that have been observed in Mamyshev regenerators are avoided [11, $14,15]$.

\section{REGENERATION OF A 160-GBIT/S SIGNAL}

In order to validate our proposed design rules, we have tested the present regenerator on a degraded 160-Gbit/s 1.5-ps pseudo-random bit sequence (PRBS). We have numerically simulated the propagation of a $2^{9}$-bit PRBS sequence of Gaussian pulses in a 50-km long transmission line made of a standard SMF-DCF dispersion management path. More precisely, the first segment consists of $50 \mathrm{~km}$ of standard single mode fiber (SMF, dispersion $\mathrm{D}=17$ $\mathrm{ps} / \mathrm{nm} / \mathrm{km}$, losses $\alpha=0.2 \mathrm{~dB} / \mathrm{km}$ and effective area $A_{\text {eff }}=80$ $\mu \mathrm{m}^{2}$ ) followed by $8.5 \mathrm{~km}$ of dispersion compensating fiber (DCF D $=-100 \mathrm{ps} / \mathrm{nm} / \mathrm{km}$, losses $\alpha=0.6 \mathrm{~dB} / \mathrm{km}$ and $A_{\text {eff }}=40 \mu \mathrm{m}^{2}$ ). The total losses of the line are fully compensated every $50 \mathrm{~km}$ by means of an Erbium doped fiber amplifier (noise figure of $4.5 \mathrm{~dB}$ ) followed by a $1-\mathrm{THz}$ flat-top optical bandpass filter so as to avoid any accumulation of spontaneous noise emission. For simplicity, TOD and polarization mode dispersion have been neglected. Figure 9(a) illustrates the resulting degraded signal after $900 \mathrm{~km}$ of propagation for an input average power of $3 \mathrm{dBm}$. We can observe a large amplitude jitter calculated to be $\pm 13 \%$ (rms value) of the average peakpower $( \pm 36 \%$ of peak-to-peak power variations have been found).

The regenerator relies on a commercially available highly nonlinear fiber (HNLF) having the following parameters : $\mathrm{D}=0.7 \mathrm{ps} / \mathrm{nm} / \mathrm{km}, \gamma=10.5 / \mathrm{W} / \mathrm{km}$. Figure $9(\mathrm{~b})$ represents the performance map of our regenerator, i.e. the calculated rms amplitude jitter at the output of the regenerator as a function of both $\xi_{L}$ (or HNLF length $\mathrm{L}_{\mathrm{HNLF}}$ ) and input average power. We can observe a relatively large area where a huge decrease of intensity fluctuations is achieved. The optimum working point was found to be $\xi_{L}=1.4$ $(\mathrm{L}=1300 \mathrm{~m})$ for an average power of $27 \mathrm{~mW}(14.2 \mathrm{dBm}$, $\left.N_{i n, 0}=1.5\right)$, in good agreement with above theoretical results. Figure 9(c), solid-line, illustrates the improvement of output rms amplitude jitter as a function of average power for the optimum distance $\xi_{L}=1.4$. It confirms that the output jitter is then greatly reduced from $\pm 13 \%$ to only $\pm 5.4 \%$. In dash-dot line, we have also computed the same simulations by taking into account for TOD $\left(0.008 \mathrm{ps} / \mathrm{nm}^{2} / \mathrm{km}\right)$ and fiber losses $(0.6 \mathrm{~dB} / \mathrm{km})$. We can observe that the curves are very close. The optimum average power is just weakly shifted by an amount corresponding to the global losses of the fiber. These results confirm the assumptions of our theoretical calculations and reinforce the efficiency of our model.

The regenerated signal is shown on Fig. 10(a) and the 
resulting eye-diagram shows a very good improvement of the signal quality with a clear eye opening. Let us note that the zeros bit slots have clearly not been improved.
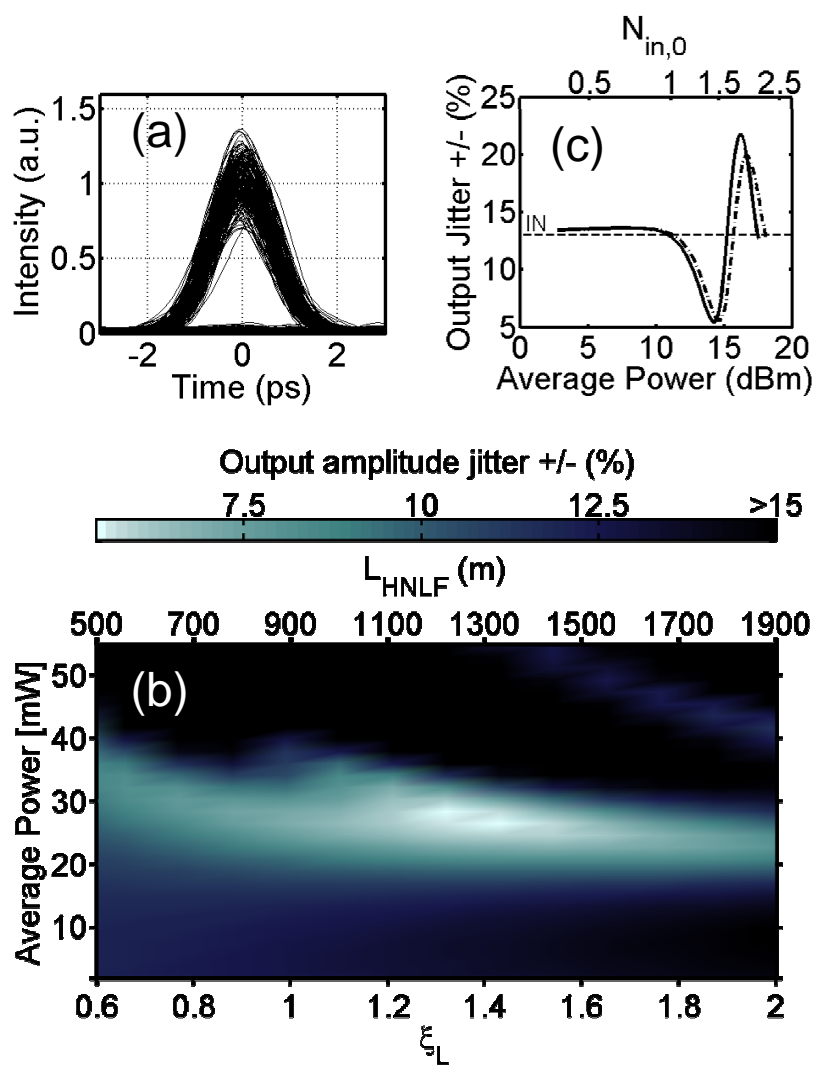

Fig. 9: (a) Input 160-Gbit/s degraded signal (b) Output rms amplitude jitter as a function of $\xi_{L}$ or $L_{H N L F}$ and average power (c) Output rms amplitude jitter as a function of average power for $\xi_{L}=1.4$. Dash-dot line, with TOD and fiber losses taken into account in the simulations.

Complementary information on the regeneration processes are provided in Fig. 10 by the various statistical distributions of the amplitude peak-power, temporal width and timing jitter. As shown in Fig. 10(b), the extreme events of peak-to-peak variations are reduced from more than $\pm 36 \%$ to $\pm 16 \%$ of the average peak-power. The statistical distribution of regenerated pulses is clearly narrower than the initial distribution (grey). The distributions of the temporal width, Fig. 10(c), or central position, Fig. 10(d), do not exhibit additional significant broadenings and are fully consistent with the discussion carried out in section IV.B.

The significant reduction of peak power fluctuations provided by our power limiter induces a high improvement of signal quality. To quantify the signal quality, we have calculated the widely used optical Q-factor [3], defined as $Q$ $=\left(P_{1}-P_{0}\right) /\left(\sigma_{1}+\sigma_{0}\right)$, with $P_{1}$ and $P_{0}$ the average level of the ones and zeros respectively at the detection instant, and $\sigma_{l}$ and $\sigma_{0}$ the corresponding standard deviations. The initial value of $Q=7$ at the input of the regenerator and after 900 $\mathrm{km}$ of propagation is significantly enhanced up to a value of $Q=15$ at the output, which allows to enhance the transmission distance in the 50-km long SMF/DCF line to $1800 \mathrm{~km}$ (distance for which $Q=6$ ), thus underlining the real efficiency of this kind of power limiter device. This improvement is indeed mainly attributed to the substantial reduction of $\sigma_{l}$ that compensates for the slight increase of $\sigma_{0}$.
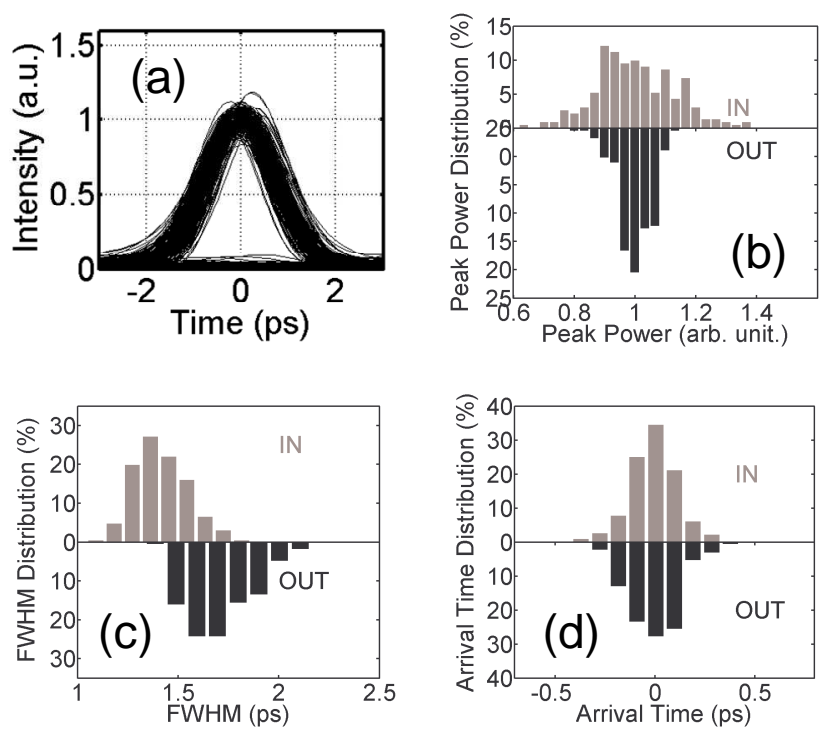

Fig. 10: (a) Output 160-Gbit/s signal for an average power of $14.2 \mathrm{dBm}$. Statistical distribution of the peak powers (b), temporal width (c) and timing jitter (d) are compared before (grey, top) and after propagation in the power limiter (black, bottom, respectively).

\section{CONCLUSION}

We have numerically studied the performance of a regenerator based on the spectral centered filtering of a signal broadened in an anomalous dispersive fiber. We have proposed general guidelines to find the optimum fiber length and initial power of the regenerator and we have quantitatively pointed out the optical limiting features of the device enabling a significant reduction of the amplitude jitter of a degraded signal. Influence of the initial level of amplitude jitter has been carefully discussed. Results have been assessed by realistic numerical simulations in the context of $160 \mathrm{Gbit} / \mathrm{s}$ signals.

Compared to existing techniques such as Mamyshev techniques, the present approach does not provide any extinction ratio enhancement of the data. However, this noticeable drawback can be quite easily overcome by an additional stage based on a saturable absorber [1, 18, 19], which have now become suitable for high speed signals up to $160 \mathrm{GHz}$ [27]. We have also shown that this approach presents several beneficial features, such as improved yield or the absence of additional timing jitter in the process. Moreover, compatibility with wavelength multiplexed signals has already been investigated [16] and can also benefit from bidirectional schemes [8].

\section{ACKNOWLEDGEMENT}

We would like to thank T. Chartier, L. Bramerie, M. Gay and T. Nguyen (Laboratoire Foton, Ecole Nationale Supérieure des Sciences Appliquées et de Technologie, France) as well as L. Provost (previously at the 
Optoelectronics Research Centre, Southampton, UK, now at Draka Comteq, Marcoussis, France) for fruitful discussion.

\section{REFERENCES}

[1] O. Leclerc, B. Lavigne, E. Balmefrezol, P. Brindel, L. Pierre, D. Rouvillain, and F. Seguineau, "Optical regeneration at $40 \mathrm{~Gb} / \mathrm{s}$ and beyond," J. Lightw. Technol., vol. 21, pp. 2779-2790, 2003.

[2] J. P. Gordon and H. A. Haus, "random-walk of coherently amplified solitons in optical fiber transmission," Opt. Lett., vol. 11, pp. 665$667,1986$.

[3] G. P. Agrawal, Fiber-Optic Communication Systems: WileyInterscience, 2002.

[4] N. J. Doran and D. Wood, "Nonlinear-optical loop miror," Opt. Lett., vol. 13 , pp. 56-58, 1988

[5] E. Ciaramella, F. Curti, and S. Trillo, "All-optical signal reshaping by means of four-wave mixing in optical fibers," IEEE Photon. Technol. Lett., vol. 13, pp. 142-144, 2001.

[6] P. V. Mamyshev, "All-optical data regeneration based on self-phase modulation effect," in European Conference on Optical Communication, ECOC'98, Institute of Electrical and Electronics Engineering, Madrid, Spain, 1998, pp. 475-476.

[7] M. Asobe, A. Hirano, Y. Miyamoto, K. Sato, K. Hagimoto, and Y. Yamabayashi, "Noise reduction of 20 Gbit/s pulse train using spectrally filtered optical solitons," Electron. Lett., vol. 34, pp. 11351136,1988

[8] L. Provost, F. Parmigiani, C. Finot, K. Mukasa, P. Petropoulos, and D. J. Richardson, "Analysis of a two-channel 2R all-optical regenerator based on a counter-propagating configuration," Opt. Express, vol. 16, pp. 2264-2275, 2008.

[9] M. Rochette, L. B. Fu, V. G. Ta'eed, D. J. Moss, and B. J. Eggleton, "2R optical regeneration : an all-optical solution for BER improvement," IEEE J. Sel. Top. Quantum Electron., vol. 12, pp. 736-744, 2006

[10] J. Leuthold, G. Raybon, Y. Su, R. J. Essiambre, S. Cabot, J. Jacques, and M. Kauer, "40 Gbit/s transmission and cascaded all-optical wavelength conversion over 1000000 km," Electron. Lett., vol. 38, pp. 890-891, 2002.

[11] T. N. Nguyen, T. Chartier, L. Bramerie, M. Gay, Q. T. Le, S. Lobo, M. Joindot, J. C. Simon, J. Fatome, and C. Finot, "Self-phasemodulation-based $2 \mathrm{R}$ regenerator including pulse compression and offset filtering for $42.6 \mathrm{Gbit} / \mathrm{s}$ RZ-33\% transmission systems," Opt. Express, vol. 17, pp. 17747-17757, 2009.

[12] T.-H. Her, G. Raybon, and C. Headley, "Optimization of pulse regeneration at $40 \mathrm{~Gb} / \mathrm{s}$ based on spectral filtering of self-phase modulation in fiber," IEEE Photon. Technol. Lett., vol. 16, pp. 200202, 2004.

[13] L. Provost, C. Finot, K. Mukasa, P. Petropoulos, and D. J. Richardson, "Generalisation and experimental validation of design rules for self-phase modulation-based 2R-regenerators," in Optical Fiber Conference, OFC 2007, Anaheim, USA, 2007, p. OThB6.

[14] C. Finot, T. N. Nguyen, J. Fatome, T. Chartier, L. Bramerie, M. Gay, S. Pitois, and J. C. Simon, "Numerical study of an optical regenerator exploiting self-phase modulation and spectral offset filtering at 40 Gbit/s," Opt. Commun., vol. 281, pp. 2252-2264, 2008.

[15] L. Provost, C. Finot, K. Mukasa, P. Petropoulos, and D. J. Richardson, "Design scaling rules for 2R-Optical Self-Phase Modulation-based regenerators 2R regeneration," Opt. Express, vol. 15, pp. 5100-5113, 2007.

[16] T. Ohara, H. Takara, A. Hirano, K. Mori, and S. Kawanishi, "40 Gb/s x 4 Channel all-optical multichannel limiter utilizing spectrally filtered optical solitons," IEEE Photon. Technol. Lett., vol. 15, pp. 763-765, 2003.

[17] T. Ohara, H. Takara, S. Kawanishi, T. Yamada, and M. M. Fejer, "160 Gb/s all-optical limiter based on spectrally filtered optical solitons," IEEE Photon. Technol. Lett., vol. 16, pp. 2311-2313, 2004.

[18] M. Matsumoto and O. Leclerc, "Analysis of 2R optical regenerator utilising self-phase modulation in highly nonlinear fiber," Electron. Lett., vol. 38, pp. 576-577, 2002.

[19] M. Gay, M. Costa e Silva, T. N. Nguyen, L. Bramerie, T. Chartier, M. Joindot, J. C. Simon, J. Fatome, C. Finot, and J. L. Oudar, "170 $\mathrm{Gbit} / \mathrm{s}$ bit error rate assessment of regeneration using a saturable absorber and a nonlinear fiber based power limiter," IEEE Photon. Technol. Lett., vol. submitted, 2009.

[20] A. Sahara, T. Inui, T. Komukai, H. Kubota, and M. Nakazawa, "40$\mathrm{Gb} / \mathrm{s} \mathrm{RZ}$ transmission over transoceanic distance in a dispersion managed standard fiber using a new inline synchronous modulation method," IEEE Photon. Technol. Lett., vol. 12, pp. 720-722, 2000.

[21] G. P. Agrawal, Nonlinear Fiber Optics, Fourth Edition. San Francisco, CA: Academic Press, 2006.

[22] A. Mecozzi, J. D. Moores, H. A. Haus, and Y. Lai, "Soliton transmission control," Opt. Lett., vol. 16, pp. 1841-1843, 1991.

[23] Y. Kodama and A. Hasegawa, "Generation of asymptotically stable optical solitons and suppression of the Gordo-Haus effect," Opt. Lett., vol. 17, pp. 31-33, 1992.

[24] M. Matsumoto, "Performance analysis and comparison of optical 3R regenerators utilizing self-phase modulation in fibers.," J. Lightw. Technol., vol. 22, pp. 1472-1482, 2004.

[25] C. Finot, B. Kibler, L. Provost, and S. Wabnitz, "Beneficial impact of wave-breaking or coherent continuum formation in normally dispersive nonlinear fibers," J. Opt. Soc. Am. B, vol. 25, pp. 19381948, 2008.

[26] D. Anderson, M. Desaix, M. Lisak, and M. L. Quiroga-Teixeiro, "Wave-breaking in nonlinear optical fibers," J. Opt. Soc. Am. B, vol. 9, pp. 1358-1361, 1992.

[27] J. Fatome, S. Pitois, A. Kamagate, G. Millot, D. Massoudre, and J. L. Oudar, "All-optical reshaping based on a passive saturable absorber microcavity device for future $160 \mathrm{~Gb} / \mathrm{s}$ applications," IEEE Photon. Technol. Lett., vol. 19, pp. 245-247, 2007. 